\title{
Evolução dos doentes com citologia oncótica alterada e colposcopia anal normal
}

\author{
Outcome of patients with anal oncotic cytology \\ alterations and normal anal colposcopy
}

\author{
SIDNEY ROBERTO NADAL ${ }^{1}$; EDENILSON EDUARDO CALORE²; THIAGO DA SILVEIRA MANZIONE³; \\ STÊNNIO PABLO MACHADO ${ }^{4}$; CARMEN RUTH MANZIONE ${ }^{5}$; VICTOR EDMOND SEID ${ }^{6}$; \\ SÉRGIO HENRIQUE COUTO HORTA ${ }^{7}$
}

\begin{abstract}
${ }^{1}$ Titular da Sociedade Brasileira de Coloproctologia. ${ }^{2}$ Professor Livre-Docente de Anatomia Patológica da Faculdade de Medicina da Universidade de São Paulo e Médico Patologista do Instituto de Infectologia Emílio Ribas. ${ }^{3}$ Titular da Sociedade Brasileira de Coloproctologia. ${ }^{4}$ Associado da Sociedade Brasileira de Coloproctologia. ${ }^{5}$ Titular da Sociedade Brasileira de Coloproctologia. ${ }^{6}$ Titular da Sociedade Brasileira de Coloproctologia. ${ }^{7}$ Titular da Sociedade Brasileira de Coloproctologia.
\end{abstract}

NADAL SR, CALORE EE, MANZIONE TS, MACHADO SP, MANZIONE CR, SEID VE, HORTA SHC. Evolução dos doentes com citologia oncótica alterada e colposcopia anal normal. Rev bras Coloproct, 2011;31(1): 71-76.

RESUMO: A citologia anal vem sendo usada para rastreamento do carcinoma anal e suas lesões precursoras nas populações de risco. Quando o raspado do canal anal mostra alterações citológicas está indicada o exame com colposcópio e ácido acético para identificar e realizar biópsia para confirmar o achado. Poucos estudos mostram o seguimento dos doentes tratados de condilomas acuminados perianais. Temos usado os métodos em associação e encontrado lesões subclínicas em metade dos doentes, cujo exame proctológico não revelava doença HPV induzida. Essas lesões são tratadas com tópicos. Entretanto, algumas citologias estavam alteradas e a colposcopia anal não revelou doença HPV induzida. O objetivo deste estudo foi observar o comportamento dessas lesões no seguimento semestral, durante 12 meses, e avaliar se a periodicidade da reavaliação foi suficiente para evitar o aparecimento das lesões de alto grau ou superior. Encontramos 58 (21\%) entre 273 doentes nessas condições. As reavaliações de 22 deles após um ano mostraram que as colposcopias permaneceram normais em $17(74 \%)$, sendo que em cinco (22\%) a citologia voltou aos padrões normais e 12 (52\%) persistiram com alterações. Os outros seis (26\%) desenvolveram lesões clínicas ou subclínicas provocadas pelo HPV. As contagens de linfócitos T CD4 dos doentes HIV-positivos foram inferiores nos doentes cujas lesões progrediram. Os resultados permitiram concluir que as alterações podem progredir ou regredir neste grupo distinto de doentes, sendo relacionada à imunidade, e que o intervalo de seis meses é suficiente para cada reavaliação.

Palavras-chave:.neoplasias do ânus; infecção por papilomavírus; carcinoma de células escamosas.

\section{INTRODUÇÃO}

O papilomavírus humano (HPV) é o mais comum dos agentes etiológicos sexualmente transmissíveis, provocando doenças na região perianal ${ }^{1}$. Embora a maioria das infecções pelo HPV não apresente manifestação clínica, $10 \%$ dos doentes desenvolverão verrugas, papilomas ou displasias ${ }^{2}$. Além disso, é descrita a possibilidade de progressão das displasias, ou neoplasias intraepiteliais anais (NIA), para carcinoma invasivo ${ }^{3}$, e a maioria ocorreria na zona de transição do canal anal ${ }^{4}$.

Outra característica das lesões HPV induzidas é a elevada incidência de recidivas. Revisando a literatu-

Trabalho realizado pelas Equipes Técnicas de Proctologia e de Anatomia Patológica do Instituto de Infectologia Emilio Ribas - São Paulo (SP), Brasil.

$\overline{\text { Recebido em: } 31 / 05 / 2010}$

Aprovado em: 14/04/2011 
ra, encontramos índices entre 10 e $88 \%$, dependendo do tratamento instituído ${ }^{5-10}$. Entretanto, é difícil diferenciar dos casos de reinfecção ${ }^{6,10}$. Há os que associaram as recidivas à presença da infecção latente pelo HPV no epitélio aparentemente normal ${ }^{11}$. Daí, a importância do seguimento por longo prazo e da pesquisa de meios terapêuticos para reduzir essa ocorrência.

A possibilidade de detecção das lesões precursoras indica que programas padronizados de rastreamento para a prevenção do câncer anal e protocolos de tratamento para NIA, em doentes de risco, deveriam ser instituídos ${ }^{12,13}$. Os esfregaços anais para citologia vêm sendo realizados ${ }^{9-11,14,15}$, com eficácia semelhante à das coletas cervicais ${ }^{16}$, e com sensibilidade oscilando entre 42 e $98 \%$ e especificidade variando de 38 a $96 \%$, quando os resultados foram comparados com os da histologia ${ }^{10,16,17}$. A maioria dos autores indica a colposcopia anal para identificar os locais mais apropriados para biópsias, quando a citologia mostra-se alterada ${ }^{11,14,18-20}$, e muitos outros recomendam-na como método de rastreamento para populações de alto risco ao carcinoma anal e seguimento após o tratamento das lesões provocadas pelo HPV ${ }^{21-24}$.

Temos utilizado os dois métodos para seguimento após o tratamento das lesões anais HPV induzidas. Neste caso, indicamos os exames 30 dias após o término do tratamento, quando não observamos doença visível, tempo suficiente para que haja cicatrização das feridas, evitando que sejam confundidas com aquelas de origem viral ${ }^{24}$. A colposcopia anal pode revelar lesões subclínicas na margem e no canal anal, e estas serão tratadas com substâncias tópicas. Posteriormente, repetimos esses métodos de diagnósticos para reavaliação. Entretanto, em alguns doentes, o exame com o colposcópio não revela lesão HPV induzida, mas a citologia do canal anal mostra-se diferente do normal, sugerindo que a doença não está completamente controlada. Não há o que tratar, nem sabemos onde realizar a biópsia dirigida para confirmar o achado. A literatura não faz referência a este fato.

O objetivo deste estudo é mostrar a evolução desses doentes com colposcopia normal e citologia alterada, na tentativa de padronizar a conduta mais adequada.

\section{MÉTODOS}

\section{Caracterização da amostra}

São 273 doentes com condilomas acuminados perianais tratados, submetidos aos exames de colposcopia anal e citologia oncótica do canal anal para seguimento, quando não havia lesões visíveis 30 dias após o término do tratamento. Quanto à infecção pelo HIV, 197 são soropositivos (189 homens e 8 mulheres) e 76 são soronegativos (38 homens e 38 mulheres). Entre os primeiros, a média etária foi 42 anos e entre os últimos foi de 28 anos.

\section{Citologia oncótica anal}

$\mathrm{Na}$ ausência de lesões clínicas, procedemos à retirada de material do canal anal para esfregaço, com escova, que introduzimos até $4 \mathrm{~cm}$ da borda anal, com movimentos de rotação ${ }^{21}$. Esfregamos a escova em lâminas de vidro, fazendo movimentos de rotação e em zigue-zague para que toda a área seja preenchida e todo o material possa entrar em contato com o vidro ${ }^{21}$. Em seguida, colhemos nova amostra, da mesma maneira. Posteriormente, acomodamos as lâminas em recipiente plástico apropriado com álcool etílico a $70 \%$ para fixação.

A coloração pela hematoxilina e eosina pode mostrar lesões intraepiteliais escamosas (SIL), que podem ser de alto (HSIL) ou de baixo grau (LSIL), conforme a quantidade de cromatina e o tamanho dos núcleos das células acometidas. Outro aspecto eventualmente observado é chamado de ASCUS, que é a sigla em inglês para células escamosas atípicas de significado indeterminado. Significa que as anormalidades encontradas são leves e de natureza incerta.

\section{Colposcopia anal (anuscopia de alta resolução)}

$\mathrm{Na}$ sequência das coletas, borrifamos ácido acético a $3 \%$ no períneo e na pele perianal e avaliamos a região após um a dois minutos, procurando pelas áreas acetobrancas, com o colposcópio convencional. Caso seja necessário e para diferenciar das alterações cicatriciais, pincelamos com azul de toluidina a $1 \%$ que cora as áreas doentes. A seguir, introduzimos o anuscópio descartável, localizando-o de maneira a expor a linha pectínea e a zona de transformação do canal anal. Aplicamos o ácido acético a 3\%, nesta região, com haste flexível e ponta coberta com algodão. Após 
a leitura, utilizamos solução iodada que cora o epitélio em castanho. As lesões HPV induzidas, como não contêm glicogênio, coram-se com menos intensidade. Colhemos biópsias exclusivamente dos locais revelados pelos corantes para avaliar o grau de NIA e/ou a presença do carcinoma.

As áreas acetobrancas podem ter aspectos pontilhados em mosaico, alterações vasculares e verrucosos (Figuras 1 a 4) Entretanto, somente o exame histológico pode certificar se a lesão observada pode ser NIA e qual o seu grau.

As lesões clínicas e as subclínicas observadas foram tratadas com substâncias tópicas. Decorrentes 30 dias do término deste tratamento e na ausência de lesões clínicas, repetimos os exames. Quando observamos colposcopia anal normal e as alterações citológicas, optamos por repetir os exames em seis meses

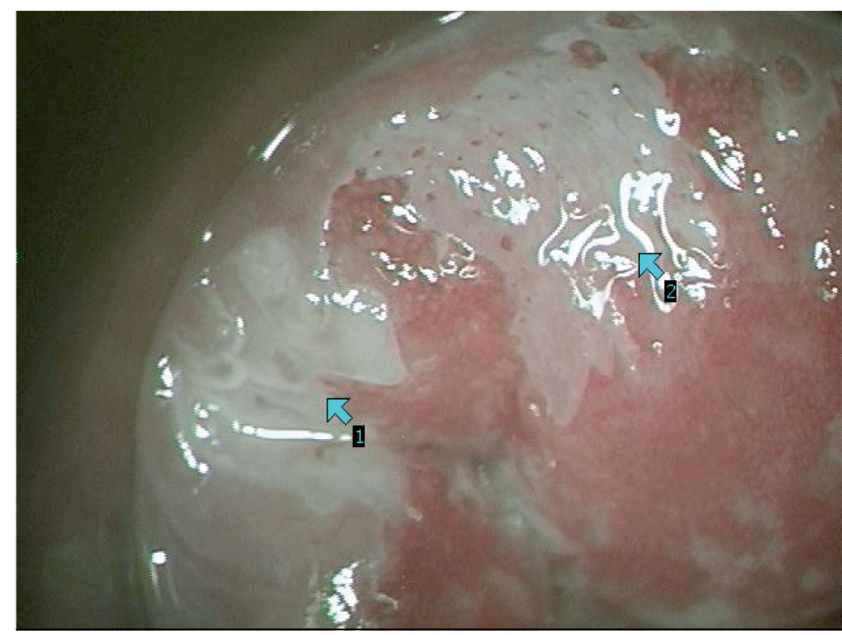

Figura 1. Pontilhado suave

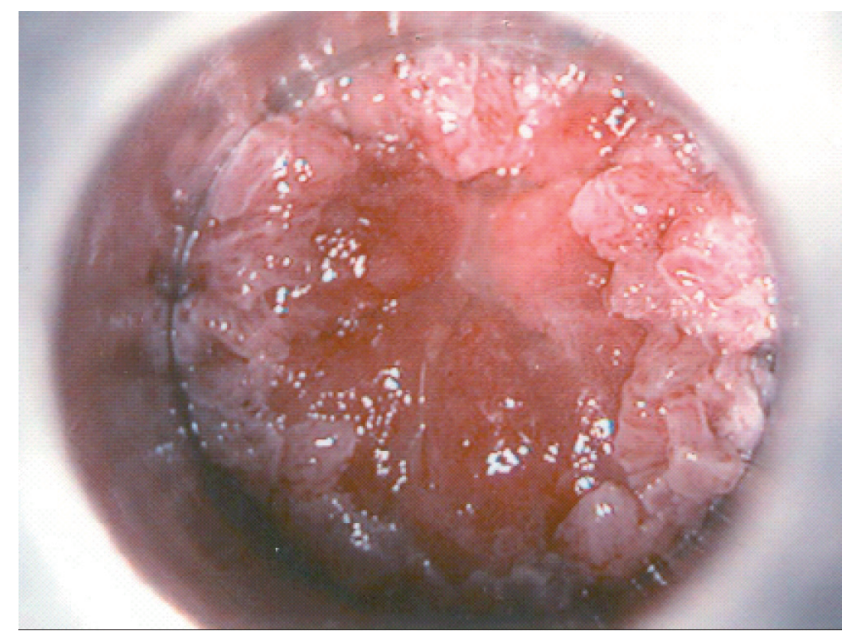

Figura 3. Lesão em mosaico e orientamos os doentes a retornarem ao ambulatório caso percebessem alguma alteração anorretal.

Utilizamos o método do qui-quadrado e o teste exato de Fischer para análise estatística dos resultados, com nível de significância de 5\%.

\section{RESULTADOS}

Dos 273 doentes seguidos desta maneira, 58 (21\%) tiveram avaliações normais pela colposcopia anal, porém, o raspado do canal anal retirado para avaliação citológica se revelou diferente do normal. Nenhum dos doentes retornou ao serviço queixandose de verrugas anais. Após 12 meses e seguimento semestral, as colposcopias permaneceram normais em $17(74 \%)$, sendo que em cinco deles (22\%) a citologia voltou aos padrões normais e $12(52 \%)$ persistiram

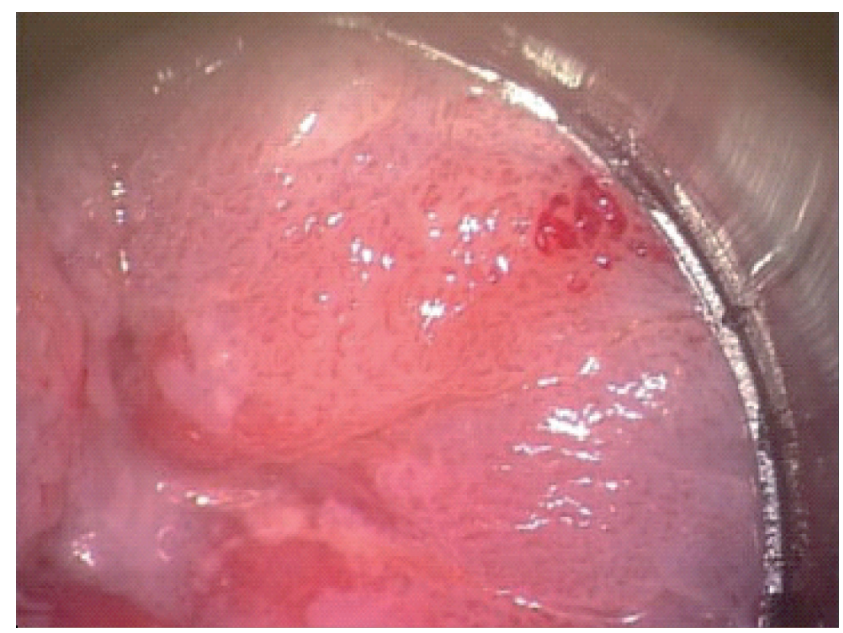

Figura 2. Alterações vasculares

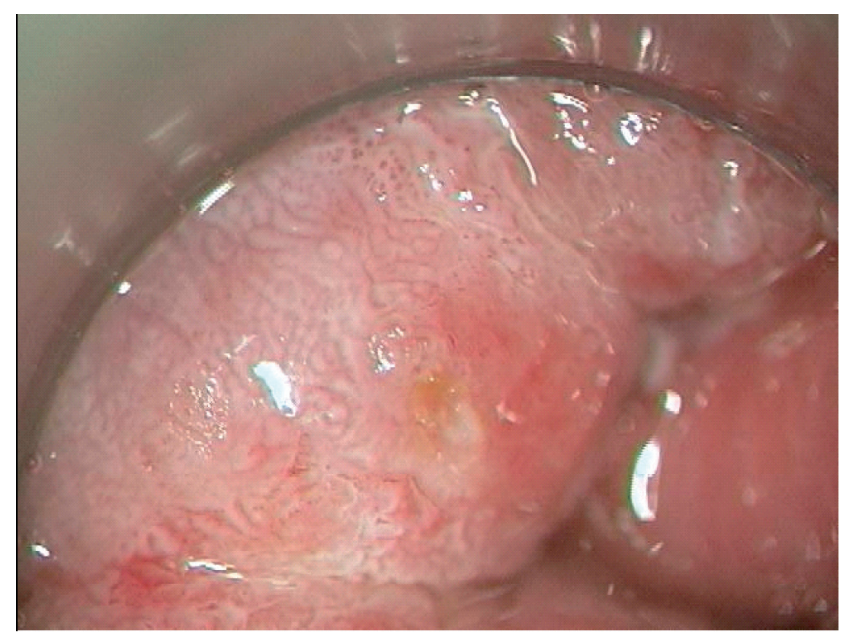

Figura 4. Lesões verrucosas 
com alterações. Os outros seis $(26 \%)$ desenvolveram lesões clínicas ou subclínicas provocadas pelo HPV. Eram 19 positivos para o HIV e quatro soronegativos, sendo que nenhum destes últimos evoluiu com lesões clínicas ou subclínicas HPV induzidas. A comparação entre os dois grupos pelo teste do qui-quadrado não mostrou diferença significante $(\mathrm{p}=0,218)$.

A contagem média dos linfócitos T CD4+, na época da primeira avaliação, dos doentes cujas citologias se tornaram normais, foi de $568,3 / \mathrm{mm}^{3}(\mathrm{dp}=225,85)$, a dos que mantiveram a citologia alterada foi de 568,7/ $\mathrm{mm}^{3}(\mathrm{dp}=328,38)$, enquanto que a dos que evoluíram com lesões clínicas ou subclínicas detectáveis pela colposcopia anal e/ou exame proctológico foi de 246,16/ $\mathrm{mm}^{3}(\mathrm{dp}=139,71)$, fato que é estatisticamente inferior às dos demais grupos $(p=0,04)$ quando comparados com aqueles cujas citologias se mantiveram alteradas e $\mathrm{p}=0,031$ para os que a citologia se normalizou.

\section{DISCUSSÃO}

Não há consenso a respeito da melhor forma de seguimento dos doentes tratados ${ }^{25}$. Utilizamos e sugerimos a padronização dos métodos da colposcopia anal e da citologia oncótica anal, como descrita, para que todos possam realizar os exames da mesma maneira e uniformizar o atendimento, visando prevenir as recidivas, o carcinoma anal e seus precursores.

Optamos pela associação da citologia ao exame com colposcópio e ácido acético, pois, em estudo anterior, notamos que a incidência das recidivas clínicas em 12 meses diminuiu de 51 para $22 \%$, embora aumentasse, de $51 \%$ para $77 \%$, a detecção das lesões HPV subclínicas perianais nos doentes tratados e que acreditávamos estarem livres da doença ${ }^{26}$. Todavia, todos estavam assintomáticos, sem perceber as lesões, mesmo que clínicas, provavelmente pelo tamanho diminuto. Encontrar doenças clínicas ou subclínicas possibilita o tratamento. Entretanto, quando apenas o exame citológico do esfregaço mostra alteração, torna-se impossível definir alguma forma de tratamento, pois, não sabermos onde estão as lesões e não consideramos correta a aplicação de tópicos ou a ressecção cirúrgica de áreas aleatórias. Pensamos que o canal anal possa ser reservatório da infecção e que num momento propício, geralmente causado pela queda da imunidade, as doenças provocadas por esse vírus possam reaparecer. Por outro lado, o colposcópio não consegue visibilizar o interior das criptas. Caso haja infecção remanescente neste local, não será detectada pelo método, mas, células com os padrões de SIL que se desprenderam podem ser captadas durante o raspado, alterando a citologia.

Não há protocolo universalmente aceito que defina qual o período para repetir a colposcopia anal e a citologia anal. Temos utilizado seis meses até que três avaliações consecutivas estejam negativas, após passarem a anuais nos doentes com risco aumentado para recidivas ou carcinoma. Esta conduta foi baseada no fato de que em até 18 meses ocorre a maioria das recidivas ${ }^{27}$. Na literatura, há autores que citaram desde intervalos de três meses, no primeiro $a^{2} o^{28}$, e de seis meses ${ }^{13}$ para seguimento, até exames anuais para rastreamento ${ }^{29}$.

Resolvemos o mesmo intervalo de seis meses para reavaliar esse grupo distinto de doentes. Após três exames consecutivos, notamos que as lesões citológicas permaneceram inalteradas em metade do grupo e desapareceram nos outros $22 \%$. Quando avaliamos as médias dos linfócitos T CD4+ séricos, não observamos diferenças. Entretanto, a média daqueles que desenvolveram doença clínica ou subclínica durante o seguimento foi estatisticamente inferior, sugerindo influência da imunidade na evolução da doença. Não encontramos citação sobre este assunto na literatura consultada, entretanto, alguns autores citaram que as recidivas são mais frequentes entre aqueles com as contagens mais baixas de linfócitos T CD $4^{30,31}$. Necessitamos aumentar este grupo de doentes para obter resultados mais fidedignos, porém, a casuística atual permite definir o período de seis meses como suficiente para reavaliação, pelo fato das lesões que evoluíram apresentarem pouca gravidade, bem como ausência de sintomas. Todavia, há autores que sugerem que a colposcopia anal seja repetida pela possibilidade de que a NIA de alto grau não tenha sido identificada ${ }^{25}$.

\section{CONCLUSÃO}

Os resultados obtidos nas condições de execução do presente estudo permitiram concluir que os doentes com colposcopia anal normal e citologia alterada, no seguimento pós-tratamento dos portadores 
dos condilomas perianais, podem evoluir tanto para normalização dos achados citológicos como evoluir para lesões clínicas e subclínicas, estas ocorrendo naqueles com menor contagem sérica dos linfócitos $\mathrm{T}$ CD4+. O intervalo de seis meses entre as avaliações foi suficiente.

ABSTRACT: Anal cytology has been used for screening the anal carcinoma and its precursors in risk populations. When anal canal smear shows cytological alterations, examination with colposcope and acetic acid is indicated to identify and perform biopsy to confirm the finding. Few studies show the follow-up of patients treated with anal HPV induced lesions. We are using both methods in association and subclinical lesions have been found in $\mathbf{5 0 \%}$ of patients, whose proctological examinations are free from HPV lesions. However, some smears have cytological alterations, despite anal colposcopy being normal. The aim of this study was to observe these lesions' behavior in a six-month follow-up, during a year, and to assess whether this periodicity of re-evaluations was enough to avoid high grade or superior lesions. We have found $58(21 \%)$ among 273 patients with these parameters. One year re-evaluations of 22 of the patients showed that anal colposcopies remained normal in $17(74 \%)$. In five $(22 \%)$, the cytology returned to normality and in 12 $(52 \%)$, the same abnormality was seen. The other six patients $(\mathbf{2 6} \%)$ developed clinical or subclinical HPV induced lesions. T CD4+ lymphocytes counts of HIV-positive patients were inferior in those whose lesions progressed. These results permitted us to conclude that cytological alterations can progress or clear in these patients, and they have close relationship with the immunity, and the sixmonth interval is enough to each re-evaluation.

Keywords: anus neoplasms; papillomavirus infections; carcinoma, squamous cells.

\section{REFERÊNCIAS}

1. Nadal SR, Manzione CR, Horta SHC. Comparison of perianal diseases in HIV-positive patients in periods before and after HAART use. Dis Colon Rectum 2008;51:1491-4.

2. Wexner SD, Smithy WB, Milson JW, Dailey TH. The surgical management of anorectal diseases in AIDS and pre-AIDS patients. Dis Colon Rectum 1986;29:719-23.

3. Safavi A, Gottesman L, Dailey TH. Anorectal surgery in the HIV+ patient: update. Dis Colon Rectum 1991;34:299-304.

4. Denis BJ, May T, Bigard MA, Canton P. Lésions anales et péri-anales au cours des infections symptomatiques par le VIH. Étude prospective d'une série de 190 patients. Gastroenterol Clin Biol 1992;16:148-54.

5. Palefsky JM, Holly EA, Gonzales J, Lamborn K, Hollander H. Natural history of anal cytologic abnormalities and papillomavirus infection among homosexual men with group IV HIV disease. J AIDS 1992;5:1258-65.

a. Manzione CR, Nadal SR, Calore EE. Postoperative followup of anal condylomata acuminata in HIV-positive patients. Dis Colon Rectum 2003;46:1358-65.

6. Goldstone SE, Winkler B, Ufford LJ, Alt E, Palefsky JM. High prevalence of anal squamous intraepithelial lesions and squamous-cell carcinoma in men who have sex with men as seen in a surgical practice. Dis Colon Rectum 2001;44:690-8.

7. Moscicki AB, Hills NK, Shiboski S, Darragh TM, Jay N, Powell K, et al. Risk factors for abnormal anal cytology in young heterosexual women. Cancer Epidemiol Biomarkers Prev 1999;8:173-8.

8. Lytwyn A, Salit IE, Raboud J, Chapman W, Darragh T, Winkler B, et al. Interobserver agreement in the interpretation of anal intraepithelial neoplasia. Cancer 2005;103:1447-56.
9. Arain S, Walts AE, Thomas P, Bose S. The Anal Pap Smear: Cytomorphology of squamous intraepithelial lesions. Cytojournal 2005;2:4.

10. Friedlander MA, Stier E, Lin O. Anorectal cytology as a screening tool for anal squamous lesions: cytologic, anoscopic, and histologic correlation. Cancer 2004;102:1926.

11. Kreuter A, Brockmeyer NH, Hochdorfer B, Weissenborn SJ, Stucker M, Swoboda J, et al. Clinical spectrum and virologic characteristics of anal intraepithelial neoplasia in HIV infection. J Am Acad Dermatol 2005;52:603-8.

12. Palefsky JM, Rubin M. The epidemiology of anal human papillomaviruses and related neoplasia. Obstet Gynecol Clin N Am 2009;36:187-200.

13. Palefsky JM, Holly EA, Hogeboom CJ, Berry JM, Jay N, Darragh TM. Anal cytology as a screening tool for anal squamous intraepithelial lesions. J Acquir Immune Defic Syndr Hum Retrovirol 1997;14:415-22.

14. Piketty C, Darragh TM, Da Costa M, Bruneval P, Heard I, Kazatchkine MD, et al. High prevalence of anal human papillomavirus infection and anal cancer precursors among HIV-infected persons in the absence of anal intercourse. Ann Intern Med 2003;138:453-9.

15. Fox PA, Seet JE, Stebbing J, Francis N, Barton SE, Strauss S, et al. The value of anal cytology and human papillomavirus typing in the detection of anal intraepithelial neoplasia: a review of cases from an anoscopy clinic. Sex Transm Infect 2005;81:142-6.

16. Papaconstantinou HT, Lee AJ, Simmang CL, Ashfaq R, Gokaslan ST, Sokol S, et al. Screening methods for highgrade dysplasia in patients with anal condyloma. J Surg Res 2005;127:8-13.

17. Vajdic CM, Anderson JS, Hillman RJ, Medley G, Grulich 
AE. Blind sampling is superior to anoscope guided sampling for screening for anal intraepithelial neoplasia. Sex Transm Infect 2005;81:415-8.

18. Varnai AD, Bollmann M, Griefingholt H, Speich N, Schmitt C, Bollmann R, et al. HPV in anal squamous cell carcinoma and anal intraepithelial neoplasia (AIN) Impact of HPV analysis of anal lesions on diagnosis and prognosis. Int $\mathrm{J}$ Colorectal Dis 2006;21:135-42.

19. Panther LA, Wagner K, Proper J, Fugelso DK, Chatis PA, Weeden $\mathrm{W}$, et al. High resolution anoscopy findings for men who have sex with men: inaccuracy of anal cytology as a predictor of histologic high-grade anal intraepithelial neoplasia and the impact of HIV serostatus. Clin Infect Dis 2004;38:1490-2.

20. Nadal SR, Calore EE, Nadal LRM, Horta SHC, Manzione CR. Citologia anal para rastreamento de lesões pré-neoplásicas. Rev Assoc Med Bras 2007;53:147-51.

21. Nadal SR, Manzione CR. Uso do colposcópio para avaliar a região perianal e o canal anal. Padronização técnica e indicações. Rev Bras Coloproct 2004;24:379-81.

22. Magi JC, Magi DAS, Reche LMC, Falavinha T, Carvalho GT. Anuscopia com exacerbação para diagnóstico de Papilomavírus Humano ano-retal na forma subclínica. Rev Bras Coloproct 2002;22:178-83.

23. Nadal SR, Manzione CR. Manejo das neoplasias intraepiteliais anais. Rev Bras Coloproct 2008;28:462-4.

24. Park IU, Palefsky JM. Evaluation and Management of Anal Intraepithelial Neoplasia in HIV-Negative and HIVPositive Men Who Have Sex with Men. Curr Infect Dis Rep 2010;12:126-33.
25. Nadal SR, Manzione TS, Formiga FB, Calore EE, Horta SHC, Seid VE, et al. Seguimento pós-tratamento dos portadores de condilomas acuminados anais com colposcópio, acido acético e citologia oncótica do canal anal. Rev Bras Coloproct 2009;29:57-8.

26. Pineda CE, Berry JM, Jay N, Palefsky JM, Welton ML. Highresolution anoscopy targeted surgical destruction of anal highgrade squamous intraepithelial lesions: a ten-year experience. Dis Colon Rectum 2008;51:829-35.

27. Stier EA, Goldstone SE, Berry JM, Panther LA, Jay N, Krown $\mathrm{SE}$, et al. Infrared coagulator treatment of high-grade anal dysplasia in HIV-infected individuals: an AIDS malignancy consortium pilot study. J Acquir Immune Defic Syndr 2008;47:56-61.

28. Herat A, Whitfeld M, Hillman R. Anal intraepithelial neoplasia and anal cancer in dermatological practice. Australas J Dermatol 2007;48:143-53.

29. Lehtovirta P, Paavonen J, Heikinheimo O. Risk factors, diagnosis and prognosis of cervical intraepithelial neoplasia among HIV-infected women. Int J STD AIDS 2008;19:37-41.

30. Massad LS, Fazzari MJ, Anastos K, Klein RS, Minkoff $\mathrm{H}$, Jamieson DJ, et al. Outcomes after treatment of cervical intraepithelial neoplasia among women with HIV. J Low Genit Tract Dis 2007;11:90-7.

Endereço para correspondência: SIDNEY ROBERTO NADAL

Rua Mateus Grou, 130

CEP: 05415-040 - São Paulo (SP), Brasil

E-mail: srnadal@terra.com.br 